\title{
Operacionalização para Portugal da Lista EU(7)-PIM para Identificação de Medicamentos Potencialmente Inapropriados nos Idosos
}

\author{
Operationalisation for Portugal of the EU(7)-PIM List for \\ Identification of Potentially Inappropriate Medicines in \\ Older Adults
}

\author{
Daniela A. RODRIGUES ${ }^{1}$, Maria T. HERDEIRO², Petra A. THURMANN ${ }^{3}$, Adolfo FIGUEIRAS ${ }^{4}$, Paula COUTINHO ${ }^{1,5}$, \\ Fátima ROQUE $\triangle 1,5$ \\ Acta Med Port 2021 Mar;34(3):194-200 - https://doi.org/10.20344/amp.13618
}

\section{RESUMO}

Introdução: Em 2015 foi publicada a lista EU(7)-PIM, que identifica medicamentos potencialmente inapropriados na população idosa e resultou de um consenso de peritos de sete países europeus. Portugal não fez parte deste grupo, pelo que na sua origem não foi adaptada para a realidade portuguesa. Com este trabalho pretendemos elaborar uma lista de medicamentos potencialmente inapropriados adaptada à realidade dos medicamentos comercializados em Portugal, através da operacionalização da lista EU(7)-PIM para a realidade nacional, avaliar a adequabilidade do seu uso na prática clínica.

Material e Métodos: Pesquisa, na base de dados Infomed do INFARMED, dos medicamentos incluídos na lista EU(7)-PIM que apresentam autorização de introdução no mercado, e análise de possíveis novos medicamentos para inclusão na lista. A ferramenta adaptada para a realidade Portuguesa foi aplicada a uma amostra de 1089 idosos polimedicados não institucionalizados, utentes de 38 unidades de cuidados de saúde primários da região centro.

Resultados: A lista final adaptada para a realidade portuguesa inclui 184 medicamentos potencialmente inapropriados (dos quais 178 são substâncias ativas, cinco são classes de medicamentos e um corresponde ao esquema terapêutico sliding scale usado nas insulinas). Dos 1089 idosos polimedicados, 83,7\% tomavam pelo menos um fármaco incluído na lista final de medicamentos potencialmente inapropriados ou pertencente a um dos grupos incluídos na lista e, em média, cada doente tomava 1,74 (IQR 1 - 2).

Discussão: Apesar da disponibilidade de fármacos no mercado ser bastante diversa, a lista EU(7)-PIM tem sido utilizada em vários países europeus. Com este estudo operacionalizamos a lista europeia para a realidade portuguesa, facilitando assim a sua aplicação na prática clínica.

Conclusão: A lista elaborada apresenta-se como uma ferramenta útil para a identificação de medicamentos potencialmente inapropriados, de fácil utilização na prática clínica e em investigação.

Palavras-chave: Idoso; Lista de Medicamentos Potencialmente Inapropriados; Portugal; Prescrição Inapropriada

\section{ABSTRACT}

Introduction: In 2015, the EU(7)-PIM List was published, which identifies potentially inappropriate medicines in older patients and resulted from a consensus of experts from seven European countries. Portugal was not part of this group, so it was not originally adapted to the Portuguese reality. With this work, we intend to elaborate a list of potentially inappropriate medicines adapted to the reality of medicines marketed in Portugal, through the operationalization of the EU(7)-PIM List for the national reality and to evaluate the adequacy of its use for clinical practice.

Material and Methods: Search, in INFARMED's Infomed database, of drugs that are included in the EU(7)-PIM List that have marketing authorization, and analysis of possible new drugs for inclusion in the list. The tool adapted to the Portuguese reality was applied to a sample of 1089 outpatient, polymedicated older patients from 38 primary care units in Central Portugal.

Results: The final PIM list adapted to the Portuguese reality includes 184 potentially inappropriate medicines (from these, 178 are active substances, five are classes of drugs, and one corresponds to the sliding scale therapeutic scheme used in insulin therapy). Of 1089 polymedicated older patients, $83.7 \%$ took at least one drug included in the final potentially inappropriate medicines list or belonging to one of the groups included in the list, and, on average, each patient took 1.74 (IQR $1-2)$.

Discussion: Even though the availability of drugs on the market is quite diverse, the EU(7)-PIM List has been used in several European countries. With this study, we operationalized the European list for the Portuguese reality, which will enable its application in clinical practice.

Conclusion: The list drawn up is a useful tool for the identification of potentially inappropriate medicines, easy to use in clinical practice and research.

Keywords: Aged; Inappropriate Prescribing; Portugal; Potentially Inappropriate Medication List

\footnotetext{
1. Unidade de Investigação para o Desenvolvimento do Interior. Instituto Politécnico da Guarda (UDI-IPG). Guarda. Portugal.

2. Departamento de Ciências Médicas e Instituto de Biomedicina. Universidade de Aveiro (iBIMED-UA). Aveiro. Portugal.

3. Department of Clinical Pharmacology. Helios University Hospital Wuppertal. University Witten/Herdecke. Wuppertal. Alemanha.

4. Centro de Investigación Biomédica en Red de Epidemiología y Salud Pública (CIBER-ESP). Santiago de Compostela. Espanha.

5. Centro de Investigação em Ciências da Saúde. Universidade da Beira Interior (CICS-UBI). Covilhã. Portugal.

$\triangle$ Autor correspondente: Fátima Roque. froque@ipg.pt
}

Recebido: 19 de fevereiro de 2020 - Aceite: 07 de setembro de 2020 - First published: 24 de novembro de 2020 - Online issue published: 01 de março de 202 Copyright @ Ordem dos Médicos 2021 


\section{INTRODUÇÃO}

O envelhecimento mundial da população é uma realidade deste século. Pela primeira vez na história, em 2018, o número de pessoas com 65 ou mais anos de idade ultrapassou o número de crianças com menos de cinco anos, sendo que as previsões para 2050 indicam que o número de adolescente e adultos jovens seja ultrapassado pelo número de idosos. ${ }^{1} \mathrm{O}$ aumento da esperança média de vida das populações é sem dúvida uma das principais conquistas alcançadas. No entanto, surge um novo desafio: conseguir que ao aumento de anos de vida correspondam anos com saúde e qualidade de vida. ${ }^{2}$

O envelhecimento está muitas vezes associado a um risco aumentado de multimorbilidades, sendo que mais de metade da população idosa sofre de várias patologias, em simultâneo, com impacto na qualidade de vida e bem-estar. ${ }^{3-5}$ Assim, a população idosa consome mais medicamentos que a população mais jovem. ${ }^{6,7} \mathrm{Um}$ estudo recente realizado em Portugal na população com 65 ou mais anos de idade, em unidades de cuidados de saúde primários, mostrou que $77 \%$ da amostra tomava mais de cinco medicamentos. ${ }^{8}$ Se, por um lado, o acesso aos cuidados de saúde e aos medicamentos, principalmente nos doentes crónicos, têm contribuído para o aumento da esperança média de vida das populações, por outro lado existe uma preocupação crescente de que muitos idosos possam estar a utilizar um número inapropriadamente elevado de medicamentos. $^{7-12}$

Com as alterações fisiológicas que ocorrem com o envelhecimento, a farmacocinética e farmacodinâmica de muitos fármacos está alterada nos idosos, modificando a resposta farmacológica esperada para alguns medicamentos. ${ }^{13-16}$ Estas alterações, associadas à presença de comorbilidades e polimedicação, tornam a população idosa mais suscetível à ocorrência de reações adversas aos medicamentos, com relevante impacto na saúde e no bem-estar. ${ }^{13,17} \mathrm{O}$ uso inadequado de medicamentos em idosos que sofrem de múltiplas doenças constitui um problema de saúde pública devido ao seu impacto na morbilidade, na qualidade de vida e no uso acrescido de recursos de saúde. ${ }^{12,15-18}$ Consideram-se medicamentos potencialmente inapropriados (PIM) em idosos aqueles cujo potencial risco é superior ao potencial benefício, especialmente quando existem alternativas disponíveis mais efetivas. ${ }^{19} \mathrm{O}$ uso de PIM é um desafio à saúde pública tendo em conta as elevadas taxas de prevalência em diversos contextos de saúde, e, têm sido associados a um maior risco de reações adversas. ${ }^{20}$

Com o objetivo de reduzir o uso de PIM nesta população têm sido desenvolvidos critérios de classificação que permitem avaliar a sua prevalência e desenvolver estratégicas para reduzir a sua utilização. ${ }^{20,21}$ Os critérios criados podem ser classificados como explícitos, implícitos ou mistos. ${ }^{21}$ Os explícitos constituem listas de medicamentos que podem ser aplicados com a mínima informação e julgamento clínico, não abordando as diferenças individuais entre os doentes, enquanto que os critérios implícitos consideram o regime terapêutico do doente e são baseados no julgamento de um profissional de saúde, sendo específicos para o doente. ${ }^{21}$

Apesar de não existirem critérios nacionais, Soares et al publicaram em 2008 a operacionalização dos critérios de Beers para Portugal. ${ }^{22}$ Os critérios de Beers são uma ferramenta de critérios explícitos desenvolvida pela Sociedade Americana de Geriatria, e têm vindo a sofrer diversas alterações ao longo dos anos, sendo a última atualização de 2019. ${ }^{23}$

Em 2015, foi criada a lista EU(7)-PIM, uma ferramenta de critérios explícitos, desenvolvida por especialistas de sete países europeus (Alemanha, Finlândia, Estónia, Holanda, França, Espanha e Suécia) que permite a identificação e comparação de PIM em idosos nestes países. ${ }^{19} \mathrm{O}$ processo de desenvolvimento da lista EU(7)-PIM teve por base a participação de vários especialistas europeus em rondas por método Delphi. A lista final é constituída por 282 PIM, sendo que 275 correspondem a substâncias ativas (código ATC - Anatomical Therapeutic Chemical Classification - de sete dígitos) e sete classes de medicamentos (código ATC de cinco dígitos), pertencentes a 55 classes terapêuticas e 34 grupos terapêuticos. Alguns critérios de inclusão de PIM foram definidos tendo em conta a dose, o tempo de uso ou o esquema terapêutico. Nesta lista podemos também encontrar duas categorias de medicamentos: a categoria A que define os PIM que devem ser evitados na população idosa em qualquer circunstância, e a categoria B que define substâncias ativas como PIM apenas no caso de certas condições clínicas ou comorbidades.

Apesar de haver poucos estudos sobre PIM em Portugal, os dados publicados mostram a importância de investigação nesta área. Não existem estudos anteriores, na população portuguesa, que utilizem a lista EU(7)-PIM, pelo que se torna fundamental a sua operacionalização para a realidade portuguesa, tendo em conta os medicamentos com autorização de introdução no mercado (AIM) nacional. Com este trabalho pretendemos elaborar uma lista de PIM adaptada à realidade dos medicamentos comercializados em Portugal, através da operacionalização da lista EU(7)-PIM, para a realidade nacional, e avaliar a adequabilidade do seu uso para identificação de possíveis PIM na prática clínica.

\section{MATERIAL E MÉTODOS \\ 1.Operacionalização da lista EU(7)-PIM para a realidade portuguesa}

1.1. Pesquisa de Medicamentos incluídos na lista EU(7)-PIM comercializados em Portugal

A partir da lista EU(7)-PIM, foram identificados todos os medicamentos incluídos na lista com AIM em Portugal, usando o código ATC, através de pesquisa na base de dados Infomed, a base de dados do INFARMED - Autoridade Nacional do Medicamento e Produtos de Saúde, IP. ${ }^{24}$ A pesquisa foi realizada em maio de 2019 e confirmada em janeiro de 2020 , para atualização dos dados nesta data. 
1.2. Identificação de possíveis PIM comercializados em Portugal, não incluídos na lista EU(7)-PIM

Dois investigadores pesquisaram medicamentos com AIM em Portugal que tivessem mecanismos de ação semelhantes aos incluídos na lista EU(7)-PIM, mas que não estivessem aí incluídos. De seguida, verificou-se se apresentavam AIM nalgum dos sete países que participaram na elaboração da lista EU(7)-PIM, através da consulta das bases de dados oficiais das agências reguladoras de cada país. A informação foi discutida entre os investigadores, e nos casos de falta de consenso foi validada por um terceiro investigador.

\section{Avaliação da aplicabilidade da lista EU(7)-PIM adap- tada à realidade portuguesa}

A lista de PIM obtida para a realidade portuguesa foi aplicada numa amostra de 1089 idosos polimedicados, cujos dados de consumo de medicamentos resultaram do projeto MedElderly [SAICT-POL/23585/2016] anteriormente desenvolvido. A amostra inclui utentes de unidades

Tabela 1 - Subtâncias ativas disponíveis em Portugal pertencentes às classes de medicamentos consideradas como PIM

\begin{tabular}{cl}
\hline Código ATC & Medicamentos potencialmente inapropriados (DCI) \\
\hline A02AB & Antiácidos contendo alumínio \\
A02AB01 & Hidróxido de alumínio \\
A02AB03 & Fosfato de alumínio \\
A02AB04 & Carbonato de di-hidróxido de alumínio e sódio \\
A02AD & Combinações e complexos contendo Al, Ca e Mg \\
A02AD02 & Magaldrato \\
A02BC & Inibidores da bomba de protões \\
A02BC01 & Omeprazol \\
A02BC02 & Pantoprazol \\
A02BC03 & Lansoprazol \\
A02BC04 & Rabeprazol \\
A02BC05 & Esomeprazol \\
A02BC06 & Dexlansoprazol \\
B03AA & Preparações orais de ferro bivalente \\
B03AA03 & Gluconato ferroso \\
B03AA07 & Sulfato ferroso \\
G03C & Estrogénios \\
G03CA03 & Estradiol \\
G03CC07 & Bazedoxifeno + estrogénios conjugados \\
G03CX01 & Tibolona \\
N02CC & Triptanos \\
N02CC01 & Sumatriptano \\
N02CC02 & Naratriptano \\
N02CC03 & Zolmitriptano \\
N02CC04 & Rizatriptano \\
N02CC05 & Almotriptano \\
N02CC06 & Eletriptano \\
N02CC07 & Frovatriptano \\
\hline & \\
\hline
\end{tabular}

de cuidados de saúde primários (faixa etária entre os 65 e os 99 anos), não institucionalizados. ${ }^{25} \mathrm{O}$ projeto obteve parecer favorável da Comissão de Ética da Administração Regional de Saúde do Centro (Parecer no 105/2017) e autorização da Comissão Nacional de Proteção de Dados (Autorização n. ${ }^{\circ} 13028 / 2017$ ). A recolha de dados sobre os medicamentos utilizados pela população idosa decorreu entre abril e agosto de 2019, em 38 unidades de cuidados de saúde primários da região centro.

\section{RESULTADOS}

\section{Operacionalização da lista EU(7)-PIM para a realida- de portuguesa}

Das 275 substâncias ativas da lista EU(7)-PIM, 173 estão disponíveis em Portugal. As sete classes de medicamentos na lista EU(7)-PIM contêm um total de 59 substâncias ativas na classificação ATC, das quais 22 estão disponíveis em Portugal (Tabela 1). A classe de medicamentos de quinina e derivados (M09AA) não inclui medicamentos com AIM em Portugal.

Foram identificadas 111 substâncias ativas como possíveis PIM a adicionar às $173 \mathrm{com}$ AIM em Portugal que constavam na lista inicial, por apresentarem mecanismos de ação semelhantes. Destas 111 substâncias ativas, apenas sete não tinham AIM em pelo menos um dos países que participaram na elaboração da lista EU(7)-PIM. Da ronda final de avaliação dos investigadores, apenas cinco substâncias ativas permaneceram como novos PIM a adicionar à EU(7)-PIM List (Tabela 2).

A lista final de PIM adaptada para a realidade portuguesa inclui 184 PIM, sendo que desses, 178 correspondem a substâncias ativas (código ATC de sete dígitos), cinco são classes de medicamentos (código ATC de quatro ou cinco dígitos) e um corresponde ao esquema terapêutico sliding scale usado nas insulinas. No Apêndice 1 (ver Apêndice 1: https://www.actamedicaportuguesa.com/revista/index.php/ amp/article/view/13618/Apendice_01.pdf) apresentamos tabela completa de PIM com justificação da classificação como PIM, bem como proposta de alternativas terapêuticas, para cada caso. Nesta lista estão presentes 47 classes terapêuticas (código ATC de quatro dígitos) e 30 grupos terapêuticos (código ATC de três dígitos).

Segundo a classificação ATC, os grupos anatómicos com maior número de PIM são o $\mathrm{N}$ - sistema nervoso $(\mathrm{n}=$ $72)$, o grupo $C$ - sistema cardiovascular $(n=29), A$ - apareIho digestivo, insulinas, antidiabéticos e glucagon (incluindo insulinas em esquema terapêutico sliding scale) $(n=28)$.

\section{Avaliação da aplicabilidade da lista EU(7)-PIM adap- tada à realidade portuguesa}

Dos 1089 idosos polimedicados da amostra em estudo, $83,7 \%$ tomavam pelo menos uma substância ativa incluída na lista final de PIM e, em média, cada doente tomava 1,74 possíveis PIM (IQR 1 - 2). Desta análise, destaca-se que $43,9 \%$ dos indivíduos da amostra toma pelo menos um medicamento do grupo dos inibidores da bomba de protões (IBP), 35,9\% toma pelo menos uma benzodiazepina 


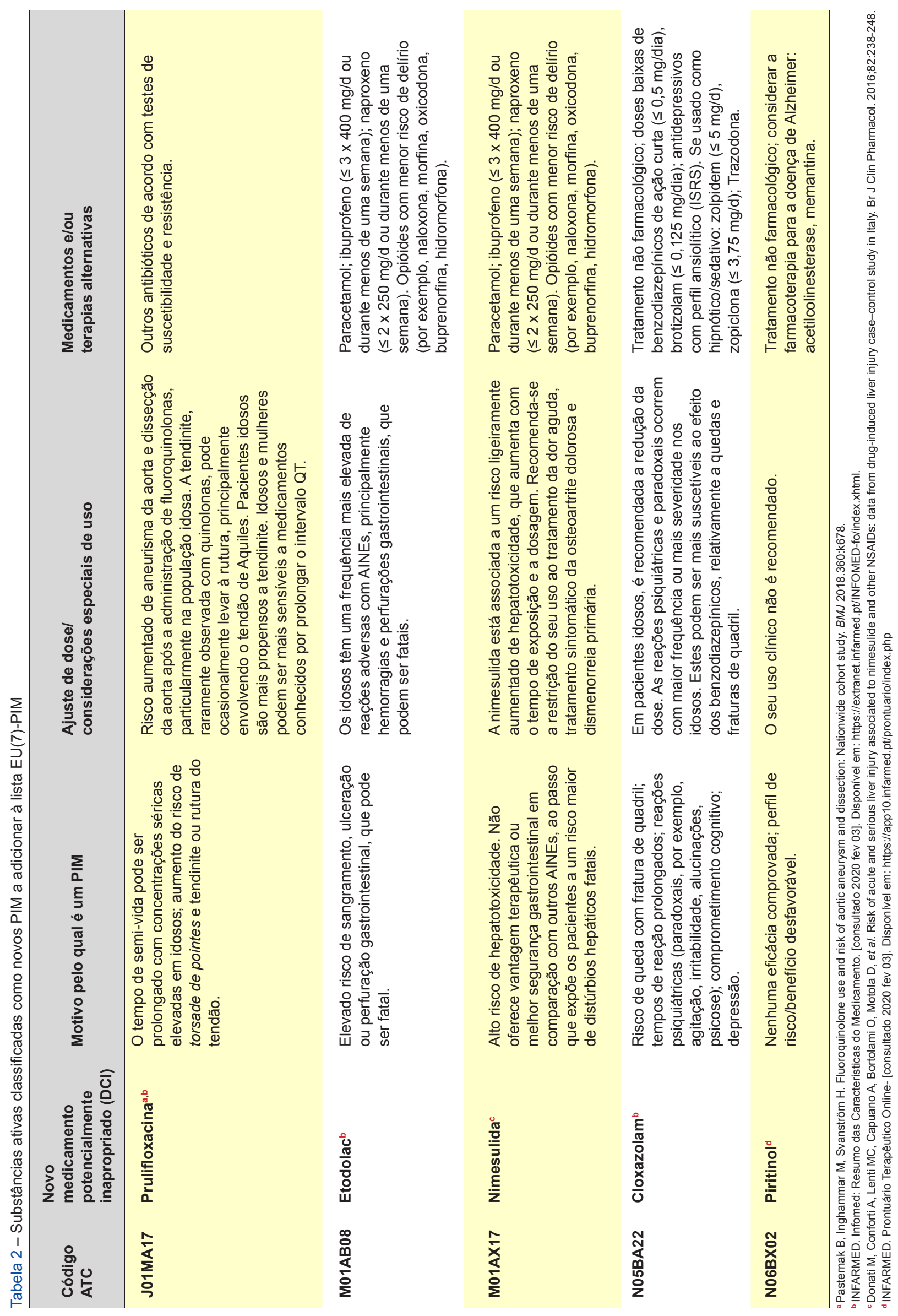


incluída na lista de PIM, e 14,4\% dos idosos da amostra está a tomar alprazolam. Na Fig. 1 apresentam-se os 10 medicamentos que são possivelmente os PIM mais prevalentes na amostra em estudo.

\section{DISCUSSÃO}

Da operacionalização da lista EU(7)-PIM para a realidade portuguesa obteve-se uma lista de critérios explícitos, dividida em três categorias: (i) substâncias ativas (código ATC de sete dígitos) com 178 PIM, (ii) classes de medicamentos (código ATC de quatro ou cinco dígitos) com cinco PIM, e, (iii) esquema terapêutico sliding scale usado nas insulinas, identificado como um PIM. Pretende-se que esta ferramenta, publicada em português e adaptada para o mercado português de medicamentos, seja útil para a prática clínica dos profissionais de saúde e para realização de estudos de investigação, permitindo a comparação com estudos e populações de outros países europeus que utilizem os mesmos critérios. Outro aspeto a salientar é que um elevado número (cerca de 39,7\%) de PIM, na lista operacionalizada, são fármacos associados a efeitos no sistema nervoso, muito provavelmente devido às propriedades anticolinérgicas destes fármacos, responsáveis por causar, no idoso, tonturas, dificuldade de concentração, perda de memória, confusão, deterioração da função cognitiva, delírio e alterações no equilíbrio. ${ }^{13,26,27}$

A disponibilidade no mercado de substância ativas é bastante diversa entre os diferentes países europeus, verificando-se, por isso, a existência de diferentes listas de PIM em função da realidade de cada país. Um estudo recente avaliou a disponibilidade de medicamentos incluídos como PIM na lista EU(7)-PIM e mostrou que o número de medicamentos com AIM, listados como PIM, é bastante diferen- te em cada país, variando entre $42,8 \%$ na Sérvia e $71,4 \%$ em Espanha. ${ }^{28}$ Num estudo belga, ${ }^{29}$ os autores mostraram que, das 275 substâncias ativas incluídas na lista original, apenas 157 estavam disponíveis no mercado. Dentro das sete classes de medicamentos, 21 substâncias ativas eram comercializadas naquele país. No mesmo estudo, os autores concluíram que a prevalência de PIM era de $72,8 \%$ de acordo com a lista operacionalizada. No nosso estudo, registámos que $83,7 \%$ dos idosos tomava pelo menos um medicamento incluído na lista operacionalizada para a realidade portuguesa. No entanto, em alguns casos, não podemos afirmar que se tratava de PIM, pois não tínhamos dados sobre o período de administração dos fármacos. Apesar de $43,9 \%$ da amostra estar a tomar IBP, não podemos concluir que estavam a tomar um PIM pois o período de administração poderia ser inferior a oito semanas. No entanto, estudos recentes têm demonstrado, que os IBP são sobreutilizados e que $25 \%$ a $70 \%$ das prescrições não têm indicação de utilização. ${ }^{30}$ As indicações de utilização como terapêutica crónica são ${ }^{31}$ : (i) doença regurgitante (DRGE) com esofagite, (ii) DRGE sem esofagite, mas com sintomas persistentes, (iii) no esófago de Barrett; iv) na úlcera péptica com episódios recorrentes e, (v) na síndrome de Zollinger-Ellison. Uma meta-análise recente ${ }^{32}$ concluiu que, apesar de o uso de IBP ter benefícios terapêuticos a curto prazo, o seu uso prolongado está associado a eventos adversos que podem ser potencialmente graves. Nos diferentes estudos incluídos nesta meta-análise foi reportada a possível associação entre o uso de IBP e doença renal crónica, pneumonia, cancro, demência, fratura, anemia, hipomagnesemia e alterações cardíacas. Assim, na população idosa, é ainda mais relevante um cuidado acrescido na monitorização da prescrição de IBP e na sua utilização

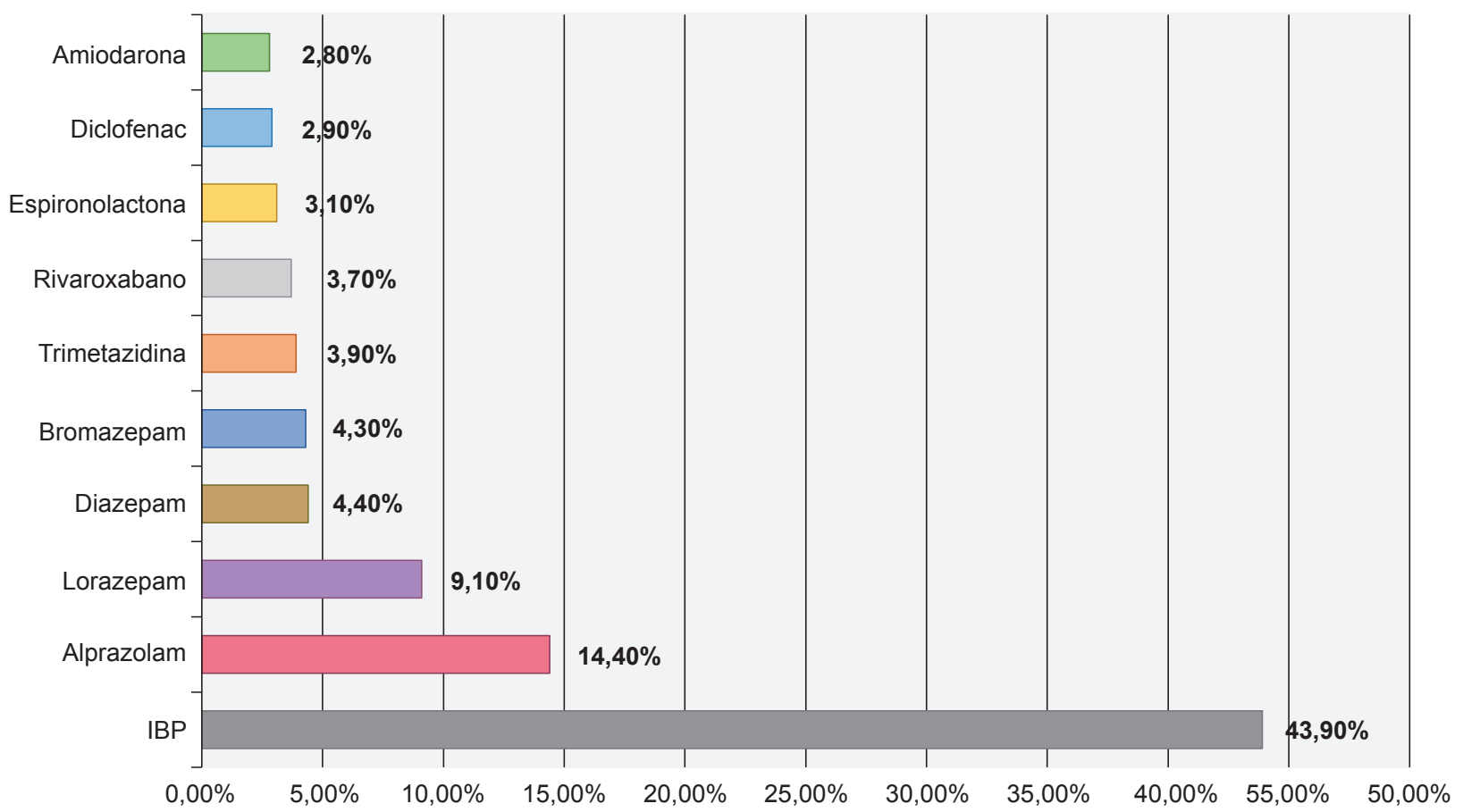

Figura 1 - Possíveis PIM mais prevalentes na amostra em estudo 
como terapêutica crónica, pelo risco acrescido de ocorrência de eventos adversos potencialmente graves.

A lista EU(7)-PIM tem sido utilizada como ferramenta de pesquisa de PIM em alguns estudos europeus. Na Finlândia, 73\% dos doentes idosos consumiram pelo menos um PIM. ${ }^{36}$ No norte da Suécia, um estudo realizado através de registos médicos de doentes idosos chegou à conclusão de que $40,9 \%$ dos doentes tinha pelo menos um PIM prescrito de acordo com a lista EU(7)-PIM. ${ }^{37}$ Na Lituânia foram encontrados $57,2 \%$ de PIM e os autores verificaram que apenas $45 \%$ dos medicamentos listados na lista EU(7)-PIM estavam disponíveis no mercado. ${ }^{38}$

Em Portugal, não existem estudos anteriores que avaliem a prevalência de PIM utilizando a lista EU(7)-PIM. No entanto, num estudo recente que utilizou os critérios de Beers, versão de 2015 , observou-se que $68,6 \%$ dos idosos incluídos na amostra em cuidados de saúde primários tomavam pelo menos um PIM e que $46,1 \%$ tomavam mais de dois. ${ }^{8}$ Outros estudos em Portugal mostraram que a prevalência de PIM em idosos institucionalizados, aplicando os critérios STOPP (Screening Tool of Older Person's Prescriptions), era de $75,4 \%,{ }^{33}$ e que em cuidados de saúde primários, utilizando os critérios de Beers versão 2012, a prevalência de PIM foi $37 \% .^{34}$ Um estudo mais antigo, realizado em farmácias comunitárias, utilizou os critérios de Beers versão de 1997 e versão de 2003, e registou uma prevalência de $27,7 \%$ e $38,5 \%$, respetivamente. ${ }^{35}$

Nos estudos de prevalência de PIM que comparam os resultados obtidos utilizando diferentes ferramentas, tem-se observado que através da aplicação da lista EU(7)-PIM, o número total de PIM identificados é superior. ${ }^{39-42}$

A utilização de critérios comuns entre países, mas com a operacionalização para a realidade de cada país, permite a criação de uma ferramenta fundamental e indispensável que poderá auxiliar os profissionais de saúde na sua prática clínica, garantindo um aumento da segurança e da eficácia terapêutica. Para além disso, este tipo de ferramentas também é importante para a realização de estudos de investigação permitindo a comparação entre países. A tabela em apêndice (ver Apêndice 1: https://www.actamedicaportuguesa.com/revista/index.php/amp/article/view/13618/ Apendice_01.pdf), com a operacionalização da lista EU(7)-PIM, constitui uma ferramenta disponível em português que poderá ser utilizada por todos os profissionais e investigadores para a realização de estudos epidemiológicos em Portugal.

Uma importante limitação deste estudo foi a amostra utilizada para avaliar a aplicabilidade da lista operacionalizada. É uma amostra não aleatória de uma região de Portugal, não permitindo a extrapolação dos resultados para outras regiões. Por outro lado, os dados disponíveis não permitiram a aplicação de todos os critérios. No entanto, o principal objetivo deste trabalho não era avaliar a prevalência de PIM em Portugal, mas sim disponibilizar uma ferramenta útil para a sua identificação, e, através deste estudo perceber que é uma ferramenta de fácil utilização na prática clínica e em investigação.

\section{CONCLUSÃO}

A exposição da população idosa a medicamentos PIM é uma realidade, com elevado consumo essencialmente de medicamentos do grupo dos IBP e do grupo das benzodiazepinas. A utilização de ferramentas de identificação de PIM, com informação sobre alternativas terapêuticas durante a prática clínica, será certamente uma mais valia na decisão de prescrição, podendo contribuir para desencorajar a prescrição de alguns destes medicamentos, conforme orientações da Direção Geral de Saúde.

Este trabalho resultou de uma análise exaustiva e rigorosa das AIMs de todos os medicamentos classificados como PIM pela lista EU(7)-PIM, com o objetivo de corresponder da melhor forma à operacionalização adaptada à realidade nacional, e assim constituir uma ferramenta útil para os profissionais de saúde. A integração desta lista, adaptada à realidade portuguesa, em sistemas de apoio à decisão clínica, constituirá uma ferramenta importante para o apoio à decisão da prescrição na população idosa e para a melhoria e segurança dos cuidados de saúde.

\section{PROTEÇÃO DE PESSOAS E ANIMAIS}

Os autores declaram que os procedimentos seguidos estavam de acordo com os regulamentos estabelecidos pelos responsáveis da Comissão de Investigação Clínica e Ética e de acordo com a Declaração de Helsínquia da Associação Médica Mundial atualizada em 2013.

\section{CONFIDENCIALIDADE DOS DADOS}

Os autores declaram ter seguido os protocolos do seu centro de trabalho acerca da publicação de dados.

\section{CONFLITOS DE INTERESSE}

Os autores declaram não ter conflitos de interesses relacionados com o presente trabalho.

\section{FONTES DE FINANCIAMENTO}

Este trabalho foi financiado pelo projeto APIMedOIder [PTDC/MED-FAR/31598/2017], através do COMPETE 2020 - Programa Operacional Competitividade e Internacionalização (POCl-01-0145-FEDER-031598), na sua componente FEDER, e por fundos nacionais através da FCT/MCTES e pelo projeto MedElderly [SAICT-POL / 23585/2016], cofinanciado pela FCT / MCTES, Portugal 2020, Centro 2020 e FEDER.

\section{REFERÊNCIAS}

1. United Nations. Department of Economic and Social Affairs. Population Division (2019). World Population Prospects 2019: Highlights (ST/ESA/ SER.A/423). Nova lorque: UN; 2019.
2. World Health Organization. Summery World Report on Ageing and Health (WHO/FWC/ALC/15.01). Luxemburgo: WHO; 2015.

3. Marengoni A, Angleman S, Melis R, Mangialasche F, Karp A, Garmen 
A, et al. Aging with multimorbidity: a systematic review of the literature. Ageing Res Rev. 2011;10:430-9.

4. Divo MJ, Martinez $\mathrm{CH}$, Mannino DM. Ageing and the epidemiology of multimorbidity. Eur Respir J. 2014;44:1055-68.

5. Arokiasamy $P$, Uttamacharya $U$, Jain $K$, Biritwum RB, Yawson AE, Wu F, et al. The impact of multimorbidity on adult physical and mental health in low- and middle-income countries: what does the study on global ageing and adult health (SAGE) reveal? BMC Med. 2015;13:178.

6. Morin L, Johnell K, Laroche ML, Fastbom J, Wastesson JW. The epidemiology of polypharmacy in older adults: register-based prospective cohort study. Clin Epidemiol. 2018,10:289-98.

7. Hosseini SR, Zabihi A, Jafarian Amiri SR, Bijani A. Polypharmacy among the elderly. J Midlife Health. 2018;9:97-103.

8. Simões PA, Santiago LM, Simões JA. Prevalence of potentially inappropriate medication in the older adult population within primary care in Portugal: a nationwide cross-sectional study. Patient Prefer Adherence. 2019;13:1569-76.

9. Opondo D, Eslami S, Visscher S, de Rooij SE, Verheij R, Korevaar $\mathrm{JC}$, et al. Inappropriateness of medication prescriptions to elderly patients in the primary care setting: a systematic review. PLoS One. 2012; 7:e43617.

10. Hedna K, Hakkarainen KM, Gyllensten H, Jonsson AK, Petzold M, Hagg S. Potentially inappropriate prescribing and adverse drug reactions in the elderly: a population-based study. Eur J Clin Pharmacol. 2015;71:152533.

11. Belfrage B, Koldestam A, Sjöberg C, Wallerstedt SM. Number of drugs in the medication list as an indicator of prescribing quality: a validation study of polypharmacy indicators in older hip fracture patients. Eur J Clin Pharmacol. 2015;71:363-8.

12. Wastesson JW, Morin L, Tan EC, Johnell K. An update on the clinical consequences of polyarmacy in older adults: a narrative review. Expert Opin Drug Saf. 2018;17:1185-96.

13. Corsonello A, Pedone C, Incalzi RA. Age-related pharmacokinetic and pharmacodynamic changes and related risk of adverse drug reactions. Curr Med Chem. 2010;17:571-84

14. Waring RH, Harris RM, Mitchell SC. Drug metabolism in the elderly: a multifactorial problem? Maturitas. 2017;100:27-32.

15. Koren G, Nordon G, Radinsky K, Shalev V. Clinical pharmacology of old age. Expert Rev Clin Pharmacol. 2019;12:749-55.

16. Thürmann PA. Pharmacodynamics and pharmacokinetics in older adults. Curr Opin Anaesthesiol. 2020;33:109-13.

17. Naveiro-Rilo JC, Diez-Juárez D, Flores-Zurutuza ML, Javierre Pérez P, Alberte Pérez C, Molina Mazo R. La calidad de vida en ancianos polimedicados con multimorbilidad. Rev Esp Geriatr Gerontol. 2014:49:158-64.

18. Davies LE, Spiers G, Kingston A, Todd A, Adamson J, Hanratty B. Adverse outcomes of polypharmacy in older people: systematic review of reviews. J Am Med Dir Assoc. 2019;21:e15385.

19. Renom-Guiteras A, Meyer G, Thürmann PA. The EU(7)-PIM list: a list of potentially inappropriate medications for older people consented by experts from seven European countries. Eur J Clin Pharmacol. 2015;71:861-75.

20. Motter FR, Fritzen JS, Hilmer SN, Paniz EV, Paniz VM. Potentially inappropriate medication in the elderly: a systematic review of validated explicit criteria. Eur J Clin Pharmacol. 2018;74:679-700.

21. Aguiar JP, Brito AM, Martins AP, Leufkens HG, da Costa FA. Potentially inappropriate medications with risk of cardiovascular adverse events in the elderly: a systematic. J Clin Pharm Ther. 2019;44:349-60.

22. Soares MA, Fernández-Llimós F, Lança C, Cabrita J, Morais JA. Operacionalização para Portugal: critérios de Beers de medicamentos inapropriados nos doentes idosos. Acta Med Port. 2008;21:441-52.

23. American Geriatrics Society Beers Criteria ${ }^{\circ}$ Update Expert Panel. Updated AGS Beers Criteria ${ }^{\circledR}$ for potentially inappropriate medication use in older adults. J Am Geriatr Soc. 2019;67:674-94.

24. INFARMED - Autoridade Nacional do Medicamento e Produtos de Saúde IP. Infomed - Base de dados de medicamentos. [consultado 2019 maio 20 a 24 e 2020 jan 31]. Disponível em: http://app7.infarmed.pt/ infomed/.

25. Gomes D, Placido Al, Mó R, Simões JL, Amaral O, Fernandes I, et al Daily medication management and adherence in the polymedicated elderly: a cross-sectional study in Portugal. Int J Environ Res Public Health. 2020;17:pii: E200.

26. Heser K, Luck T, Röhr S, Wiese B, Kaduszkiewicz H, Oey A, et al. Potentially inappropriate medication: association between the use of antidepressant drugs and the subsequent risk for dementia. J Affect Disord. 2018;226:28-35.

27. Cross AJ, George J, Woodward MC, Ames D, Brodaty H, Wolfe R, et al. Inappropriate medication, anticholinergic burden, and mortality in people attending memory clinics. J Alzheimers Dis. 2017;60:349-58.

28. Fialová D, Brkić J, Laffon B, Reissigová J, Grešáková S, Dogan S, et al. Applicability of EU(7)-PIM criteria in cross-national studies in European countries. Ther Adv Drug Saf. 2019;10:1-22.

29. Wauters M, Elseviers M, Azermai M, Vander Stichele R. Availability and actual use in the Belgian market of potentially inappropriate medications (PIMs) from the EU(7)-PIM list. Eur J Clin Pharmacol. 2016;72:243-5.

30. Jaynes $M$, Kumar $A B$. The risks of long-term use of proton pump inhibitors: a critical review. Ther Adv Drug Saf. 2018;10:1-13.

31. Direção-Geral da Saúde. Norma DGS: Supressão Ácida: Utilização dos Inibidores da Bomba de Protões e das suas Alternativas Terapêuticas. Lisboa: DGS; 2011.

32. Islam MM, Poly TN, Walther BA, Dubeyd NK, Ningrum DN, Shabbir SA, et al. Adverse outcomes of long-term use of proton pump inhibitors: a systematic review and meta-analysis. Eur J Gastroenterol Hepatol. 2018;30:1395-405.

33. da Costa FA, Periquito C, Carneiro MC, Oliveira P, Fernandes Al, Cavaco-Silva P. Potentially inappropriate medications in a sample of Portuguese nursing home residents: Does the choice of screening tools matter? Int J Clin Pharm 2016;38:1103-11.

34. Eiras A, Teixeira MA, González-Montalvo Jl, Castell MV, Queipo R, Otero Á. Consumo de medicamentos en mayores de 65 años en Oporto (Portugal) y riesgo de prescripción de medicamentos potencialmente inapropiados. Aten Primaria. 2016;48:110-20.

35. De Oliveira Martins S, Soares MA, Foppe Van Mil JW, Cabrita J. Inappropriate drug use by Portuguese elderly outpatients - effect of the Beers criteria update. Pharm World Sci. 2006;28:296-301.

36. Bobrova V, Heinämäki J, Honkanen $O$, Desselle $S$, Airaksinen $M$, Volmer D. Older adults using multi-dose dispensing exposed to risks of potentially inappropriate medications. Res Soc Adm Pharm. 2018;9:1102-6.

37. Sönnerstam E, Sjölander M, Gustafsson M. An evaluation of the prevalence of potentially inappropriate medications in older people with cognitive impairment living in Northern Sweden using the EU(7)-PIM list. Eur J Clin Pharmacol. 2017;73:735-42.

38. Grina D, Briedis V. The use of potentially inappropriate medications among the Lithuanian elderly according to Beers and EU(7)-PIM list - a nationwide cross-sectional study on reimbursement claims data. J Clin Pharm Ther. 2017;42:195-200.

39. Muhlack DC, Hoppe LK, Stock C, Haefeli WE, Brenner H, Schöttker B. The associations of geriatric syndromes and other patient characteristics with the current and future use of potentially inappropriate medications in a large cohort study. Eur J Clin Pharmacol. 2018;74:1633-44.

40. Almeida TA, Reis EA, Pinto IV, Ceccato MD, Silveira MR, Lima MG. Factors associated with the use of potentially inappropriate medications by older adults in primary health care: an analysis comparing AGS Beers, EU(7)-PIM List, and Brazilian Consensus PIM criteria. Res Soc Adm Pharm. 2019;15:370-7.

41. Stojanović $M$, Vuković $M$, Jovanović M, Dimitrijević S, Radenković M. Potentially inappropriate medications in nursing home residents: a comparison of two approaches. Eval Health Prof. 2020;163278719900653.

42. Muhlack DC, Hoppe LK, Saum KU, Haefeli We, Brenner H, Schöttker B. Investigation of a possible association of potentially inappropriate medication for older adults and frailty in a prospective cohort study from Germany. Age Ageing. 2019;49:20-5. 